\section{Evaluation of a weekly flushing policy for central venous catheters}

\author{
By Karin Olson
}

\section{Abstract}

The standard nursing protocol for central venous catheter (CVC) care at the Cross Cancer Institute was recently changed from daily flushing with 100 units $/ \mathrm{ml}$ heparinized normal saline to weekly flushing with 10 units $/ \mathrm{ml}$ heparinized normal saline. The purpose of this study was to determine whether there was any change in the $C V C$-related infection rate or CVC occlusion rate following this change, and to evaluate the cost implications. Data were collected on 39 patients (43 lines). Only one $(2 \%)$ line became occluded and the $C V C$ infection rate was $8 \%$ for non-bone marrow transplant participants and $18 \%$ for bone marrow transplant participants. On the basis of these findings, weekly flushing was retained as part of the standard nursing protocol for $\mathrm{CVCs}$.

Despite the use of central venous catheters (CVCs) for more than a decade, the research base for nursing management of these lines remains incomplete. One of the areas of concern is flushing frequency. Two complications thought to be related to flushing frequency are infection and occlusion.

\section{Literature review \\ Infection}

Infection rates associated with CVC daily flushing protocols are reported to vary between $5.6 \%$ (Raaf, 1985) and $46 \%$ (Al-Sabai, 1987). While the comparison of infection rate data between studies is difficult, due to variation in factors such as the definition of infection and the nature of the population, Weightman, Simpson and Speller (1986) suggested that, since many CVC line infections were attributable to contaminated catheter caps, a reduction in daily manipulation might lead to a reduction in infections.

In their study of cancer patients, Kelly, Dumenko, McGregor and McHutchion (1990) defined an infection as a positive catheter culture or positive catheter tip culture. If cultures were negative, but upon line removal a patient's febrile condition improved, that patient's line was also counted as infected. They found that, when CVCs were flushed weekly, $10 \%$ of non-BMT and $30 \%$ of BMT patients developed infections. These findings compared favourably with previously published studies of cancer patients, where rates varied from $12 \%$ (Pessa, 1985) to $55 \%$ (Al-Sabai, 1987). Kelly et al. also found, however, that their rate of line infections per 100 patient days was considerably below those previously reported for daily flushing protocols ( $7 \%$ for non-BMT and $25 \%$ for BMT patients). The infection rates for daily flushing protocols previously published range between $21 \%$ (Pessa and Howard, 1985) and 53\% (Kappers-Klunne and Degener, 1985). While there are some limitations in the above studies related to factors such as the differences in the definition of infection, the findings suggest that by decreasing the manipulation of CVCs from daily to weekly, the infection rate will also decrease.

\section{Occlusion}

There is limited information in the current literature regarding the association between the rate of occlusion and flushing frequency.
Studies in this area are difficult to compare, since some investigators considered a line to be occluded if blood could not be withdrawn but a flush could be carried out, while others only considered a line to be occluded if it would not flush. Petersen, Clift, Hickman, Sanders, Meyers, Kelleher and Buckner (1986) conducted a study in which CVCs were flushed twice daily with $6 \mathrm{ml}$ of heparinized saline. They stated that clotting and other malfunctions were the cause of catheter removal in $6.7 \%$ of the patients. Cassidy, Zayko, Bron, Reilly, Peitzman and Steed (1987) reported the formation of a fibrin sheath in $57 \%$ of occluded catheters in their study, whereas Raaf (1985) reported that only $3.9 \%$ of the lines (in 698 patients) infused, but did not allow withdrawal of blood. Reilly, Steed and Ritler (1984) reported that $15 \%$ of the lines in their study were removed due to occlusion. Kelly, Dumenko, McGregor and McHutchion (1990) considered a line occluded if they were unable to withdraw blood or could not flush it. They reported that $10 \%$ of their lines became occluded, but that patency was regained in all cases. These findings suggest that changing the flushing frequency from daily to weekly does not appreciably alter the frequency of occlusion.

Based on the above findings, we recently changed our standard nursing protocol from daily flushing with $3 \mathrm{ml} /$ lumen of heparinized normal saline $(100: 1)$, to weekly flushing with $3 \mathrm{ml} /$ lumen of heparinized normal saline (10:1). In this article, we report the results of an evaluation conducted to determine whether there was any subsequent alteration in our CVC-related infection or occlusion rates, and the cost implications of this practice change.

\section{Method}

The clinical outcomes of interest in this project were occlusion and septicemia. Occlusion was defined as the inability to flush and obtain blood return. Septicemia was defined as oral temperature above $38.5 \mathrm{C}$ and positive blood cultures (same organism in samples drawn from CVC and peripheral vein). The line of one patient who had an oral temperature above $38.5 \mathrm{C}$, but negative blood culture, was removed due to unresolved infection. Since her temperature returned to normal shortly after the line was removed, this was also counted as a CVC-related septicemia.

\section{Sample}

The sample consisted of all patients whose double or triple lumen Quinton Raaf catheters were inserted at our hospital between June 1991 and May 1992. Patients were followed through their entire admission, which included both inpatient and outpatient admissions for some patients.

\section{Data collection}

All CVCs were inserted in the operating room by a surgeon under sterile conditions. All chart data were collected by an experienced oncology nurse who had worked extensively with bone marrow transplant patients. Infection data were obtained from lab reports. Since physicians must write an order for the medications used to clear occluded lines, occlusion data was obtained by checking the physician order sheet and the nursing notes. Compliance with the new weekly flushing protocol was checked by conducting telephone interviews with $20 \%$ sample of study participants. The telephone interviews were conducted by an undergraduate nursing student.

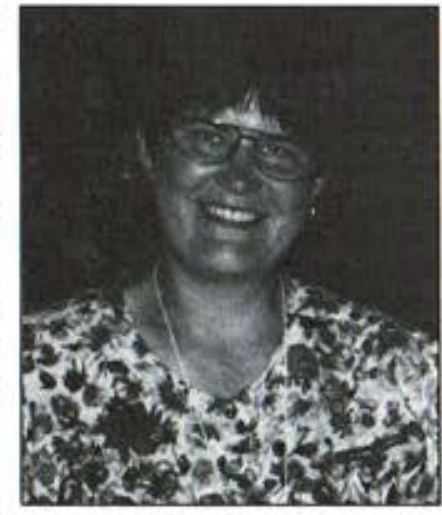

Karin Olson

Karin Olson, $\mathrm{RN}, \mathrm{PhD}$, is coordinator, nursing research, at the Cross Cancer Institute, Edmonton, Alberta, and past chair of the CANO research committee. 


\section{Findings}

Data from 39 patients (43 lines) were collected. Of these, 25 were non-bone marrow transplant (BMT) patients and 14 were BMT patients. As shown in Table One, $61 \%$ of the lines remained completely patent. Of the remaining lines, flushing was easy but blood could not be withdrawn from $23 \%$; this problem later resolved without any intervention, indicating that the tips of these lines were temporarily resting against the wall of a vein. In an additional $12 \%$, flushing was easy but streptokinase was required to establish blood return, indicating the likely presence of a fibrin sheath at the end of the catheter. One line $(2 \%)$ could not be flushed because it was interstitial. Only one line ( $2 \%)$ appeared to be truly occluded, but no action was taken, since it was already scheduled for removal.

When the daily flushing protocol was in place, the occlusion rate was estimated by the nursing staff to be approximately $10 \%$. The difference between the $10 \%$ occlusion rate during the daily flushing and the $2 \%$ occlusion rate during the weekly flushing was tested using a single sample test of proportions. The $\mathrm{Z}$ was significant at the 0.038 level. These findings should be received cautiously, since the occlusion rate for the daily flushing protocol was an estimate. However, it is unlikely that the occlusion rate under the weekly flushing protocol is significantly worse than under the daily flushing protocol. We are of the view that the lower occlusion rate under the weekly flushing protocol is likely due to fewer entries into the line, hence less trauma to the vein.

We did not have any data on our own CVC-related septicemia rate during the daily flushing protocol, but as can be seen in Table Two, our CVC-related septicemia rate for weekly flushing with non-BMT patients of $8 \%$ is close to that reported by Kelly et al. (1992). We attribute this finding to less frequent entries into the line and, therefore, fewer opportunities to introduce contaminants. Our CVC-related infection rate among BMT patients was $18 \%$. This figure is lower than that reported by Kelly et al. and, in our view, is probably related to our use of gauze rather than occlusive dressing. The exit sites of participants in the study conducted by Kelly et al. were covered with occlusive dressings.

Table One: Patency in 43 CVCs flushed weekly with $3 \mathrm{ml}$ of heparinized normal saline $(10: 1)$

$\begin{array}{lrr}\text { Line characteristic } & \text { N } & \% \\ \text { Lines remaining patent } & 26 & 61 \\ \text { Lines that could only be flushed } & & \\ \quad \text { Positional } & 10 & 23 \\ \quad \text { Required streptokinase } & 5 & 12 \\ \text { Lines occluded } & 1 & 2 \\ \text { Lines interstitial } & 1 & 2\end{array}$

\section{References}

Al-Sabai, M., Harden, E., Faskin, R., Johnson, G., \& Padmos, A. (1987). The value of prophylactic antibiotics during the insertion of long-term indwelling silastic right atrial catheters in cancer patients. Cancer, 60, 1891-1895.

Cassidy, F., Zayko, A., Bron, K., Reilly, J., Peitzman, A., \& Steed, D. (1987). Noninfectious complications of long-term central venous catheters: Radiologic evaluation and management. American Journal of Radiology, 149, 671-675.

Kappers-Klunne, M. \& Degener, J. (1985). Complications from long-term indwelling central venous catheters, with special reference to infections. Netherland Journal of Medicine, 28, 192-196.

Kelly, C., Dumenko, L., McGregor, E., \& McHutchion, E. (1992). A change in flushing protocols of central venous catheters. Oncology Nursing Forum, 19(4), 599-605.
In the second phase of the evaluation, a data collector telephoned a $20 \%$ simple random sample $(n=9)$ of patients whose CVCs were inserted during the study period. Six patients maintained weekly flushing. Four of these had no problems and the remaining two had some difficulty initiating the flush but were able to instill the remaining flushing solution with no further difficulty. Two of the nine patients experienced flushing problems that required an increase in flushing frequency. Of these, one patient had a blockage that required the use of streptokinase. This line was then maintained by flushing it every two days. The second patient had some initial difficulty flushing his line and subsequently maintained it by flushing it every two to three days. The last patient flushed the catheter every time the dressing was changed, just for convenience.

\section{Cost implications}

According to figures presented by Kelly, Dumenko, McGregor and McHutchion (1992) the cost to flush a double line daily is approximately $\$ 8.26 /$ week, or $\$ 429.52 /$ year. The cost to flush a double line weekly would be approximately $\$ 1.18 /$ week or $\$ 61.36 /$ year . Assuming an average length of line placement of 186 days, the cost of weekly flushing for the duration of the line would be $\$ 31.35(186 / 7 \mathrm{x}$ 1.18 ) while the cost of daily flushing would be $\$ 219.48(186 / 7 \times 8.26)$, with a per patient savings of $\$ 188.13$. Given that we follow approximately 30 adult patients with new $\mathrm{CVCs} /$ year, the savings associated with this proposed change would be $\$ 5,644.00$.

\section{Conclusion}

Based on these findings, all patients are taught to flush their CVCs weekly, being sure to establish positive pressure in the line when the flush is finished. Patients who experience flushing difficulty that does not spontaneously clear are advised to increase flushing frequency to once every two to three days. A study to evaluate the use of a weekly flush with bacteriostatic normal saline is currently underway.

\section{Acknowledgement}

The author acknowledges the assistance of Linda Read Paul and Joanne Pollard, who were the data collectors on this project. The project was funded by a Job Enhancement Grant from the Ministry of Health, Province of Alberta.

Table Two: Septicemia in 43 CVCs flushed weekly with $3 \mathrm{ml}$ of heparinized normal saline (10:1)

Lines

BMT

Non-BMT

\begin{tabular}{rr}
\multicolumn{2}{c}{ Sepsis } \\
N & $\%$ \\
3 & 18 \\
2 & 8
\end{tabular}

\begin{tabular}{cr}
\multicolumn{2}{c}{ No sepsis } \\
N & $\%$ \\
14 & 82 \\
24 & 92
\end{tabular}

Pessa, M. \& Howard, R. (1985). Complications of Hickman Broviac catheters. Surgery, Gynecology and Obstetrics, 161, 257-260.

Petersen, F., Clift, R., Hickman, R., Sanders, J., Meyers, J., Kelleher, J., \& Buckner, C. (1986). Hickman catheter complications in marrow transplant recipients. Journal of Parenteral and Enteral Nutrition, 10(1), 58-62.

Raaf, J.(1985). Results from use of 826 venous access devices in cancer patients. Cancer, 55, 1313-1321.

Reilly, J., Steed, D., \& Ritler, P. (1984). Indwelling venous access catheters in patients with acute leukemia. Cancer, 53, 219-223.

Weightman, N., Simpson, L., \& Speller, D. (1986). Source of infection in Hickman catheters. Journal of Clinical Pathology, 39(9), 1046. 